\title{
Fluorescein Sodium-Guided Surgery of Malignant Brain Tumors: History, Current Concepts, and Future Projects
}

\author{
Karl-Michael SCHEBESCH, Alexander BRAWANSKI, Christoph HOHENBERGER, Julius HÖHNE \\ University Medical Center Regensburg, Department of Neurosurgery, Germany
}

\section{ABSTRACT}

Fluorescein sodium (FL)-guided resection has become an important and beneficial treatment method for malignant brain tumors. FL-guided resection improves the rate of gross total resection in high-grade gliomas (HGG) and cerebral metastases (CM). FL sensitively visualizes the disruption of the blood-brain barrier in the area surrounding malignant lesions, similar to contrast-enhanced T1-weighted MR sequences.

This review of the current literature summarizes the history of FL in neurosurgery from 1946 until today. We discuss the molecular mechanism of FL accumulation in cerebral malignant tumors and provide an overview of the current practice of using FL and applying a dedicated surgical microscope filter. Additionally, we outline and discuss ongoing trials and future projects.

KEYWORDS: Fluorescence-guided surgery, Fluorescein sodium, High-grade glioma, Cerebral metastases, YELLOW $560 \mathrm{~nm}$ filter, Malignant brain tumor

\section{INTRODUCTION}

Malignant brain tumors such as high-grade gliomas (HGG) or cerebral metastases are devastating diseases. Modern comprehensive treatment of HGG consists of resection, chemotherapy, and radiation. The extent of resection (EOR) has been repeatedly proven to be one of the most important predictors of overall survival (OS), progression-free survival (PFS), and neurological outcome $(21,25,31,43)$. Thus, consensus exists that -whenever safely possible- neurosurgical resection should aim at completely removing contrast-enhanced tissue in the preoperative T1-weighted magnetic resonance (MR) sequence.

Accordingly, the EOR is known to significantly increase the efficacy of any adjuvant treatment of both recurrent HGG and cerebral metastases by prolonging OS and PFS and improving neurological outcome $(20,39,53)$.

Since the invention of the surgical microscope in the 1960s, both the accuracy and efficacy of neurosurgical performance has been steadily increased by improved magnification and illumination methods. Furthermore, the introduction of imageguidance (neuro-navigation and intraoperative ultrasonography) facilitated the identification of tumor margins in the 1990s, thus improving the quality of resection of malignant brain tumors.

Since the results of a phase-III trial confirmed a significantly higher rate of gross-total resection (GTR) with 5-ALA in comparison to resection under white light (48), fluorescence-guided surgery with 5-aminolevulinic acid (5-ALA) in combination with a dedicated surgical microscope filter (BLUE400, Carl Zeiss Meditec, Germany) has extended the neurosurgical armamentarium for HGG.

However, the use of 5-ALA has also several distinct disadvantages. 5-ALA is expensive, and its use must still be approved by the American Food and Drug Administration (FDA). Furthermore, exposure to light after 5-ALA injection may cause severe porphyria and other skin reactions that require complex patient management. The most important disadvantage for neurosurgeons, however, is that the use of 
5-ALA under filtered light is limited and cumbersome. The surgical field appears dark, while tumor tissue is stained purple. Coagulation and the dissection of vessels or non-tumorous tissue are either complicated or unfeasible. The interpretation of fluorescence remains largely subjective, particularly at the tumor margins, necessitating permanent switching from bluefiltered to white light. Furthermore, the therapeutic value of 5-ALA has been shown for HGG but not for other malignant cerebral tissue, such as cerebral metastases (30).

Fluorescein sodium (FL), a fluorophore that has been wellknown in ophthalmic surgery for almost five decades, has been increasingly used and researched in neuro-oncologic surgery since the late 1990s. Based on several publications by the active group of Toshihiko Kuroiwa $(23,24)$, the intraoperative use of $F L$ in combination with a dedicated surgical microscope filter has become a matter of great scientific and clinical interest around the world. Today, neurosurgical centers are increasingly equipped with the technical requirements for the intraoperative use of FL. Recently, the First National European Pharmacologic Agency has approved FL for use in neurosurgery (Agenza Italiana del Farmaco'AIFA', determina n.905/2015, 15 luglio 2015).

In this review, we provide the history of FL in neurosurgery and give an overview on current trials and future concepts for using FL for the treatment of malignant brain tumors.

\section{MOLECULAR MECHANISM and BLOOD-BRAIN BARRIER}

\section{Fluorescein Sodium (FL)}

Spiro [isobenzofuran-1(3H)'9'[9H]xanthene]-3-one, 3'6'-dihydroxydisodium salt- $\mathrm{C}_{20} \mathrm{H}_{10} \mathrm{O}_{5} \cdot 2 \mathrm{Na}$ - with the established name 'Fluorescein sodium'(FL) is the sodium salt of fluorescein. Its molecular weight is 376.27 (http://www.scbt.com/ datasheet-206026-fluorescein-sodium-salt.html).

The synthetic organic compound FL was discovered by Adolf von Baeyer in 1871. So far, FL has been widely used as a fluorescent tracer in technical chemistry, microscopy, serology, and forensics. Poured into water, the powder colored red or dark orange immediately turns yellow. Exposure to special (yellow-filtered) light with a wavelength of approximately 560 nanometers $(\mathrm{nm})$ significantly increases the color intensity (http://chemfinder.cambridgesoft.com).

\section{Blood-Brain Barrier (BBB) and Cerebral Lesions}

The blood-brain barrier is characterized by tight interendothelial cell junctions and adjacent astrocyte end feet separated by basal lamina surrounding the endothelium (26). An intact BBB defends the central nervous system (CNS) from intrusion of foreign substances and maintains CNS homeostasis, including ionic and fluid balances (18). Abbott et al. could show that the balance of this microenvironment is disrupted by cerebral lesions such as gliomas. The disturbance of the integrity leads to (increased) vascular permeability and 'leakage of fluid into the brain parenchyma which results in high pressure and brain edema (1).
In 2013, Nduom et al. analyzed the BBB both histopathologically and immunohistochemically with regard to metastatic and primary malignant neoplasms. The authors could show that areas of contrast-enhancement (Gadolinium, Gd) in the magnetic resonance imaging (MRI) significantly corresponded to a broken-down BBB in the cerebral tissue surrounding the tumor. Conversely, intratumoral vasculature in low-grade gliomas and other non-enhancing lesions maintained an intact BBB (35).

In a very recent review of the literature entitled 'Novel delivery methods bypassing the blood-brain and blood-tumor barriers', Hendricks et al. outlined the surgical implications for fluorophores such as FL that serve as a very sensitive marker for a BBB breakdown in the surrounding area of HGG and cerebral metastases (CM) (18).

\section{- EVOLUTION of FLUORESCENCE-GUIDED NEUROSURGERY}

\section{'From Bench to Bedside' (1871 to 1961)}

The use of FL in technical chemistry has an astonishingly broad spectrum. Since its discovery in 1871, FL has served as a contrast dye and fluorophore in a wide range of different applications.

During World War II, air force pilots carried small containers of $\mathrm{FL}$ and in case of being shot or having to parachute into water, a vivid marking was produced that was seen over long distances (Advanced Organic Chemistry, Bahl 1977, page 1266).

In 1962, FL was used to dye the Chicago River green on St. Patrick's day (The Story Behind Dyeing the River Green. Greenchicagoriver.com). Today, FL aids leak detection in subsea oil and gas pipelines and other subsea infrastructures (https://en.wikipedia.org/wiki/Fluorescein).

The first application of FL in human medicine dates back to 1946. Patients subjected to laparotomy for gastric cancer received FL injections to distinguish between malignant and normal tissues. Dissection was done under violet light for improved illumination as previously described by Danckwortt in 1934 (Danckwortt P, Luminiszenz-Analyse im filtrierten ultravioletten Licht. Leipzig: Akademische Verlagsgesellschaft, 1934).

The US-American surgeon George E. Moore pioneered FL-guided surgery for malignant tumors. He was the first to summarize and publish the results of his own series in Science, by reporting on 46 patients mostly with tumors of the gastrointestinal tract, brain, and spinal cord (33). One year later, he outlined the diagnostic value of FL for the intraoperative detection of brain tumors under ultraviolet light in his publication 'The clinical use of fluorescein in neurosurgery' in the Journal of Neurosurgery. However, Moore had no explanation for the increased concentration of FL in malignant tissue (34).

In July 1961, Harold Novotny and David L. Alvis published the paper 'A method of Photographing Fluorescence in Circulating 
Blood in the Human Retina' in Circulation (36). FL was injected in the antecubital vein and, after 30 seconds, 'striking luminescence appeared in the retinal vessels'. Undoubtedly, this discovery had been the start of establishing $\mathrm{FL}$ as a valuable dye for retinal vessels, resulting in its worldwide acceptance and uncountable consecutive applications in ophthalmic surgery (40).

\section{FL in Modern Neurosurgery (1998-2015)}

High dose FL and white light: In 2003, Jun Shinoda et al. published a series of 105 patients with glioblastoma of whom 32 had been operated on under white light after a high-dose injection of FL (20 mg/kg bodyweight). A GTR rate of $84.4 \%$ could be achieved in the FL group in contrast to only $30.1 \%$ in the non-FL group. The fluorescent areas corresponded well to areas of gadolinium uptake in the contrast-enhanced preoperative MRI. Because of this finding, the authors concluded that FL demarcated areas of BBB breakdown similar to that of gadolinium in T1. In deeply stained regions, typical glioblastoma features were confirmed histologically. In faintly stained specimens, 'less dense tumor cells' were found, and 'less dense tumor cells but no endothelial proliferation' was confirmed in non-fluorescent areas. In contrast to 5-ALA, the authors did not report any 'bleaching' after the administration of FL and thus concluded that high-dose FL is beneficial in glioma surgery, even without any special illumination (47).

These findings were confirmed 5 years later by Koc et al. 80 patients with HGG WHO IV were included, 47 of whom were operated on under xenon white light illumination after i.v. injection of high-dose $\mathrm{FL}(20 \mathrm{mg} / \mathrm{kg}$ bodyweight). Compared to the non-FL group ( $\mathrm{n}=33$ ), a significantly higher rate of GTR was achieved in the FL group ( $83 \%$ versus $55 \%$ ). Consequently, the authors concluded that the use of $\mathrm{FL}$ in HGG was 'simple and safe' and increased the rate of complete resection. However, overall survival did not differ significantly between the two groups (22).

Another prospective trial comparing the effect of high-dose FL (15-20 $\mathrm{mg} / \mathrm{kg}$ bodyweight) under white light in patients with HGG was presented by Chen et al. in 2012. 10 patients were assigned to the $\mathrm{FL}$ group, and 12 patients served as control. Significant differences in GTR rates were observed: $80 \%$ in the FL group versus $33 \%$ in the control group, resulting in a significant difference in PFS (7.2 months versus 5.4 months). No difference in postoperative neurological performance was observed (7).

In 2013, Liu et al. presented the results of a prospective study concerning the combination of MR-diffusion tensor imaging (DTI) and intraoperative tumor-staining with $\mathrm{FL}$ ( $\mathrm{n}=83$; fixed doses: $10 \mathrm{ml}$ of $10 \% \mathrm{FL}$ in adults and $0.3 \mathrm{ml} / \mathrm{kg}$ bodyweight in children) in patients with glioma in eloquent areas. Both GTR and paralysis rates differed significantly between the 'experimental' FL group and the control group ( $80.4 \%$ versus $40.7 \%$, and $25.0 \%$ versus $66.7 \%$, respectively). Consequently, the authors concluded that FL-guided resection increases the rate of GTR and improves quality of life compared to traditional glioma surgery (28). The summarized characteristics of these studies and the GTR rate are presented in Table IA.

Low-dose FL and dedicated filters: For neurosurgical purposes, members of the Toshihiko Kuroiwa group from Japan were the pioneers who revitalized the investigation into $\mathrm{FL}$ to improve the quality of resection of malignant brain tumors. In a report on 10 patients with HGG, the authors described the modification of a conventional surgical microscope that had been equipped with a xenon lamp 'Superlux'300, a BP 450-490 nm excitation filter (Zeiss, Germany), and a KODAK Wratten No.12 barrier filter $(500-530 \mathrm{~nm}) .8 \mathrm{mg}$ of FL per kg of bodyweight were injected after durotomy, and tumor dissection started about 20 minutes afterwards. In all patients, intraoperative fluorescence seemed to correspond well with the areas of gadolinium-enhancement in the preoperative MRI. An $80 \%$ rate of complete resection was confirmed by postoperative computed tomography (CT) or MRI. The authors concluded that fluorescent staining was due to the lack of normal blood-brain barrier function and that the combination of i.v. FL-injection and microscope filter maybe useful in glioma surgery (23).

Based on these encouraging findings, the same group published their personal experiences with $\mathrm{FL}$ in 1999, concluding that 'this method is very useful for exhibiting regions that lack the blood-brain barrier, where images are enhanced on CT scans and MR images' (24).

Table IA: High Dose and White Light

\begin{tabular}{lccccc}
\hline Authors & Year & No. of patients & Dose of FL & Tumor entity & GTR or complete resection \\
\hline Shinoda et al. & 2003 & 105 & $20 \mathrm{mg} / \mathrm{kg}$ & HGG (WHO IV) & $84.4 \%$ \\
\hline Koc et al. & 2008 & 80 & $20 \mathrm{mg} / \mathrm{kg}$ & HGG (WHO IV) & $83.0 \%$ \\
\hline Okuda et al. & 2010 & 36 & $20 \mathrm{mg} / \mathrm{kg}$ & CM & $86.1 \%$ \\
\hline da Silva et al. & 2010 & 6 & $1000 \mathrm{mg}$ & skull base tumors & $100 \%$ \\
\hline Chen et al. & 2012 & 22 & $15-20 \mathrm{mg} / \mathrm{kg}$ & LGG, HGG (WHO II-IV) & $80.0 \%$ \\
\hline Liu et al. & 2013 & 83 & n/a & LGG, HGG (WHO I-IV) & $80.4 \%$ \\
\hline da Silva et al. & 2014 & 5 & $1000 \mathrm{mg}$ & Meningiomas & $100 \%$ \\
\hline
\end{tabular}

HGG: High grade glioma, LGG: Low grade glioma, CM: Cerebral metastases, GTR: Gross-total resection, FL: Fluorescein sodium. 
Roberto Rey-Dios and Aaron Cohen-Gadol were the first to report on the dedicated 'YELLOW $560 \mathrm{~nm}$ filter'(Y560) integrated in the 'Pentero 900' surgical microscope (Carl Zeiss Meditec, Germany). This special filter had been designed to detect FL fluorescence in the range of $540 \mathrm{~nm}$ to $690 \mathrm{~nm}$. The authors crafted their technical description with three case presentations including cerebral metastasis, arteriovenous malformation (AVM), and aneurysm. The simple and safe application of the microscope filter, the 'high magnification in deep exposures with excellent detail' and the easy handling of the fluorescent dye FL made the authors conclude that FL fluorescence appeared to be 'a promising technology with potential advantages over the fluorophores currently in use' (41).

The first feasibility study on the use of the Y560 filter in combination with i.v. FL-injection in a large patient cohort was published in 2013 in Acta Neurochirurgica by the authors of this review. In 35 patients with intracranial lesions of different etiology, this new technology was found to be practical and safe, allowing consistent microsurgical dissection in the filter mode and improved visualization of tumorous tissue through the microscope oculars (44). A photograph of the intraoperative setup of the microscope with the characteristic blue beam of light due to the activation of the $Y 560 \mathrm{~nm}$ filter is presented in Figure 1.

In the same issue of Acta Neurochirurgica, the Italian group of Francesco Acerbi published the preliminary results of the FLUOGLIO study. 12 patients with HGG had been enrolled and analyzed: the rate of complete resection was $75 \%$, and histological analysis confirmed a high accuracy of using fluorescein for tumor identification. The calculated rates for sensitivity and specificity were $91 \%$ and $100 \%$ (2).

In 2014, the Indianapolis group of Aaron Cohen-Gadol focused on the accuracy of tissue diagnosis during stereotactic needle biopsy after administration of $3 \mathrm{mg}$ of FL per kg of bodyweight. 26 specimens from 6 patients with suspected HGG were obtained. The calculated rates for sensitivity and specificity were $79 \%$ and $100 \%$ (42).

Additional data on the quality of fluorescence staining in HGG were provided by Acerbi et al. in 2014. Again, preliminary data from the FLUOGLIO trial $(n=29)$ showed sensitivity and specificity rates for FL of $94.0 \%$ and $89.5 \%$. The GTR rate

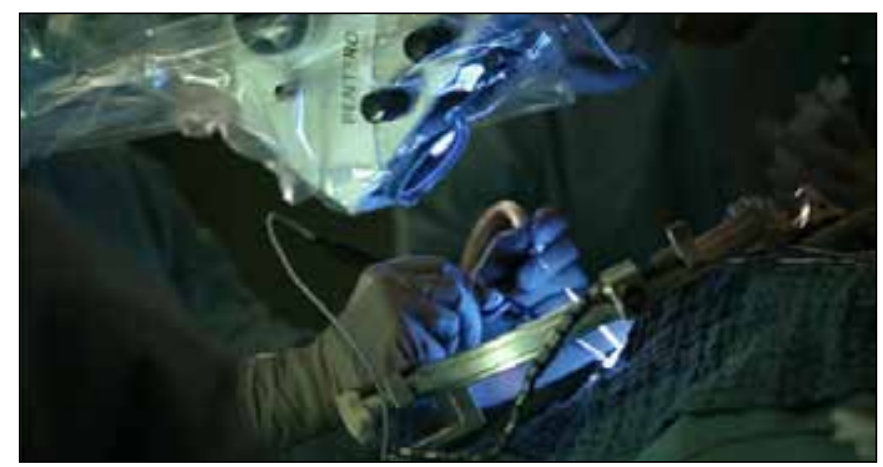

Figure 1: Intraoperative setting after application of the $Y 560 \mathrm{~nm}$ filter: note the characteristic blue beam of light. was $80 \%$, and, in the remaining patients, the mean EOR was $92.6 \%$ (3). Mitchel Berger commented on this paper outlining the great potential of FL-guided resection of HGG in an editorial and emphasized the necessity of further large prospective series (5).

In 2015, the Indianapolis group reported on 12 prospectively enrolled patients with HGG. All patients were operated on by means of the FL-guided technique under the Y560 filter. The rate of complete resection was $100 \%$-as proven by early postoperative contrast-enhanced $\mathrm{MRI}-$ and intraoperative neuro-navigation (contrast-enhanced T1 weighted sequences) corresponded well to the fluorescing areas. According to the authors, this finding strongly supports the impression that Gd-enhanced MRI is equivalent to FL fluorescence in HGG. Furthermore, the authors provided an analysis of FL accuracy and reported sensitivity and specificity rates of $82.2 \%$ and 90.9\% (11).

The summarized characteristics of these studies and the GTR rate are presented in Table IB. The calculated sensitivity and specificity rates of $\mathrm{FL}$ in combination with the $\mathrm{Y} 560 \mathrm{~nm}$ filter are presented in Table II.

Figure 2 shows an intraoperative photograph after activation of the Y560 nm filter in a patient with recurrent HGG. This photograph was chosen to illustrate the clear-cut border between fluorescing tumor and non-fluorescing brain tissue.

\section{IN VITRO EXPERIMENTS and LABORATORY INVESTIGATION Of FL}

Ichioka et al. presented experimental work on FL in 2004. Human serum-albumin-conjugated fluorescein was compared to conventional i.v. FL-injection in rodents. In contrast to conventional $\mathrm{FL}$, albumin-conjugated fluorescein was significantly brighter under the microscope, and fluorescence lasted significantly longer. Furthermore, albumin-conjugated fluorescein was entrapped in the tumor, whereas FL was also found in damaged BBB in other tissue (19).

In 2014, Martirosyan et al. provided experimental data on the useof a confocal endomicroscope in combination with various fluorophores in a rodent-glioma model $(n=25)$. Among the evaluated dyes were FL, 5-ALA, acridine orange, acriflavine, and cresyl violet. Within 5 minutes after the injection, tumor areas with disrupted BBB were characterized by extravasation of FL. Additionally, cytoarchitectural characteristics could be exactly determined, and qualitative fluorescence intensity was in the highest range. Thus, the authors concluded that handheld confocal microscopy with FL represents a useful tool for real-time in vivo histopathological assessment and visualization of malignant cerebral lesions (32).

In 2015, the Indianapolis group presented a combination of a small prospective clinical trial including 12 patients with HGG with a histopathological study consisting of in vitro and in vivo experiments in glioma-infiltrated mice. This elaborated investigation showed that 'fluorescein demarcation of gliomainvaded brain is the result of distribution of fluorescein into the extracellular space, most likely as a result of an abnormal 
Table IB: Low Dose and Filter

\begin{tabular}{lccccc}
\hline Authors & Year & No. of patients & Dose of FL & Tumor entity & GTR or complete resection \\
\hline Kuroiwa et al. & 1998 & 10 & $8 \mathrm{mg} / \mathrm{kg}$ & HGG (WHO III, IV) & $80.0 \%$ \\
\hline Schebesch et al. & 2013 & 35 & $200 \mathrm{mg}$ & LGG, HGG (WHO I-IV), CM & . \\
\hline Rey-Dios & 2014 & 6 & $3 \mathrm{mg} / \mathrm{kg}$ & HGG (WHO IV) & n/a \\
\hline Acerbi et al. & 2014 & 20 & $5-10 \mathrm{mg} / \mathrm{kg}$ & HGG (WHO III, IV) & $80.0 \%$ \\
\hline Schebesch et al. & 2015 & 30 & $200 \mathrm{mg}$ & CM & $83.3 \%$ \\
\hline Diaz & 2015 & 12 & $3 \mathrm{mg} / \mathrm{kg}$ & HGG (WHO IV) & $100 \%$ \\
\hline
\end{tabular}

HGG: High grade glioma, LGG: Low grade glioma, CM: Cerebral metastases, GTR: Gross-total resection, FL: Fluorescein sodium.

Table II: Calculated accuracy of FL in HGG, Confirmed by Histopathology

\begin{tabular}{lccc}
\hline Authors & Year & Sensitivity & Specificity \\
\hline Rey-Dios et al. & 2014 & $79.0 \%$ & $100 \%$ \\
\hline Acerbi et al. & 2014 & $94.0 \%$ & $89.5 \%$ \\
\hline Diaz et al. & 2015 & $82.2 \%$ & $90.9 \%$ \\
\hline
\end{tabular}

blood brain barrier'. No FL uptake into tumor cells was observed in the rodent model, but the histopathological workup of the infiltration zone in the clinical setup showed a high FL specificity rate of $90.9 \%$ and a FL sensitivity rate of $82.8 \%$. Consequently, the authors concluded that $F L$ is a marker for an affected or disrupted BBB in HGG (11).

This very important article by Roberto Jose Diaz was commented on by Peter E. Fecci in an editorial in the Journal of Neurosurgery. In a very elaborate way, the authors of the editorial indicated that FL may help increase the EOR, hereby positively influencing PFS and OS. However, more experimental and clinical data are required (15).

In Figures $3 \mathrm{~A}-\mathrm{C}$, the same specimen is shown under different visualization modes. In Figure $3 A$, white light is applied to a specimen without any visible tumor tissue. In Figure $3 B$, the Y560 nm filter is activated, and the yellow fluorescing spot demarcates the suspicious area. HE staining is shown in Figure $3 \mathrm{C}$, clearly depicting the tumorous area similar to the fluorescing spot.

\section{USE of FL in NON-GLIOMA LESIONS}

In 2010, the indication for fluorescence-guided surgery with FL for brain tumors was extended to cerebral metastases and skull base tumors.

\section{Cerebral Metastases (CM)}

Okuda et al. presented a retrospective analysis of 36 patients with $\mathrm{CM}$ from various primary cancers, who underwent microsurgery under white light after the injection of high-dose FL (20 mg/kg bodyweight). Complete resection was achieved in 31 of 36 patients, and 20 patients were not assigned to adjuvant whole brain radiotherapy (WBRT). Out of this group,

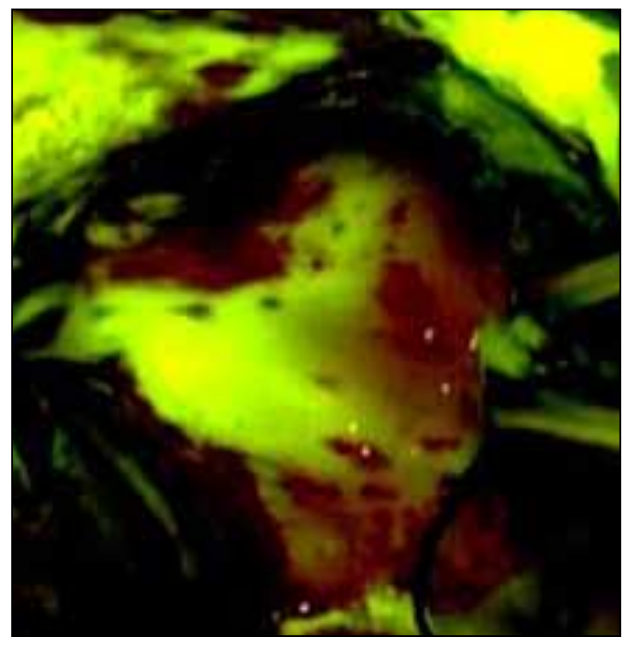

Figure 2: HGG (glioblastoma multiforme) under Y560 illumination; the white line demarcates the clear-cut border between the fluorescing tumor and the non-fluorescing tissue.

only 4 patients developed local recurrence during the followup (median 8 months). The authors concluded that -compared to conventional surgery- use of FL may decrease recurrence rates (38).

In 2015, the authors of this review published the first report on the use of FL under the Y560 filter in 30 patients with CM. Bright fluorescent staining was found in $90 \%$ of the patients, and the GTR rate was $83.3 \%$ (45). In an editorial to this report, Gempt and Meyer supported our impression that FL may be beneficial in CM surgery. However, large prospective studies are mandatory to confirm the efficacy of FL (16).

The different microscopic views are shown in Figures 4A, B. Figure 4A shows white light and Figure 4B fluorescent staining that exactly demarcates the localization of subcortical metastasis.

\section{Meningiomas and Skull Base Tumors}

Da Silva et al. reported on a prospective mini-series of 6 patients with skull base tumors (1 acoustic neuroma, 3 meningiomas, 1 craniopharyngioma, 1 pituitary adenoma), who were operated on after an injection of $1000 \mathrm{mg}$ of $20 \%$ FL under white light illumination. Particularly in meningiomas, dural enhancement was clearly visible that may have been due to dural infiltration of the tumors. However, this issue was not investigated further $(8,9)$. 


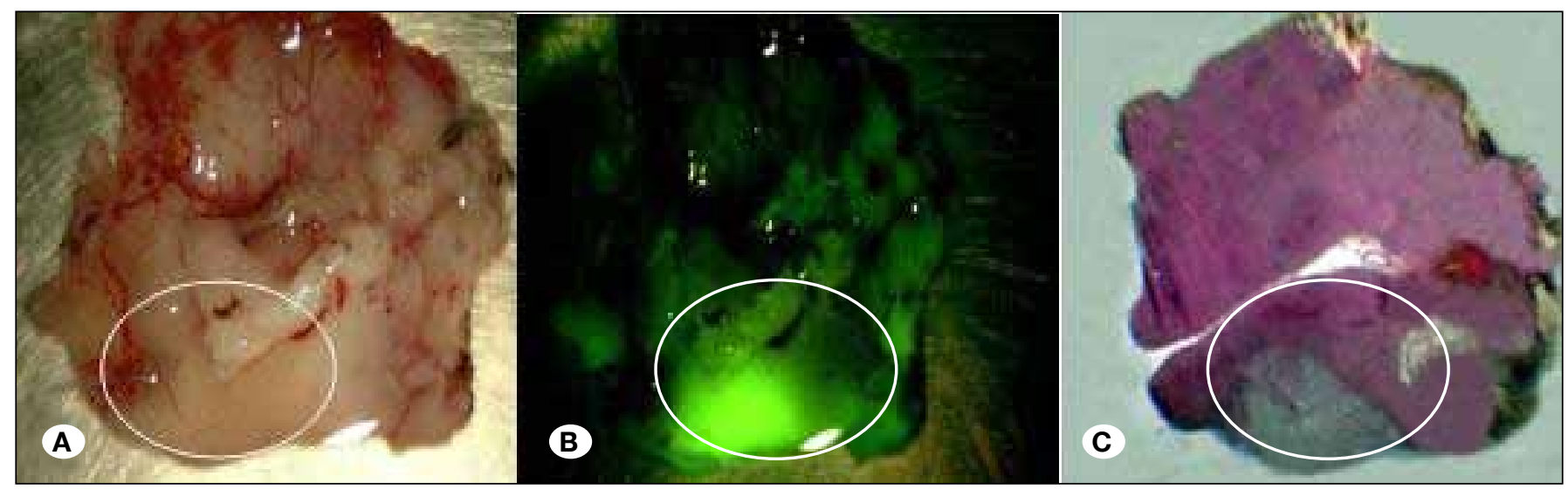

Figure 3: Specimens with tumor spots (glioblastoma multiforme); the white circle demarcates the tumorous area. A) White light, B) Y560 illumination, C) H\&E staining.
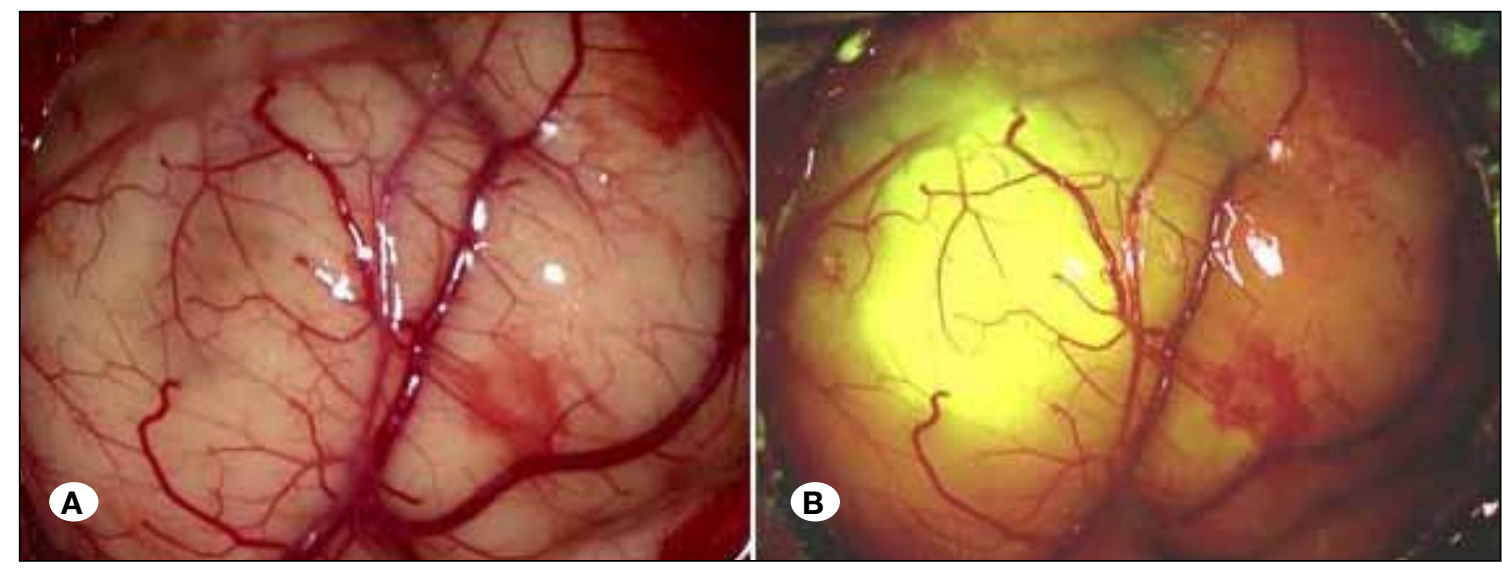

Figure 4:

Subcortical metastasis (breast cancer) A) White light, B) Y560 illumination.
The same group provided a report on 5 frontal convexity meningiomas that had been resected after the injection of $1000 \mathrm{mg}$ of $20 \% \mathrm{FL}$. The 'dural tail', as indicated by MRI, corresponded well with the intraoperative findings, and histopathological analysis confirmed the correlation between fluorescence and tumor infiltration (10).

Although the reports about the use of FL in meningiomas and other (benign) skull base tumors were promising, prospective studies with larger cohorts are necessary to confirm the efficacy and benefit of FL-guided surgery in these entities.

\section{Primary Central Nervous System Lymphoma (PCNSL)}

The first description of the use of FL in PCNSL surgery was provided by Okuda et al. High-dose FL was injected after the opening of the dura. Fluorescent staining was observed in stereotactic needle biopsies as well as endoscopically. The authors concluded that FL was effective in the diagnosis and surgery of PCNSL (37).

In cooperation with the Italian group of Francesco Acerbi, the authors of this review retrospectively analyzed the data of 7 patients who had the diagnosis of PCNSL confirmed by the final histological examination. The preoperative contrastenhanced MRI of these patients showed distinct contrastenhanced lesions strongly suspicious of CM or HGG. Because the grade of intraoperative fluorescence staining was excellent in all cases, we concluded that FL under the Y560 filter can be very helpful in identifying small and deeply located PCNSL lesions (unpublished data) (46).

\section{COMPREHENSIVE REVIEWS of the LITERATURE on FL-GUIDED SURGERY}

Since 2013, 4 reviews on fluorescence-guided neurosurgery in general have been published. These reviews comprehensively discuss the variety of fluorophores and their possible applications, advantages, and disadvantages in neuro-oncologic and vascular neurosurgery: Behbahaninia et al. in 2013 (4), Li et al. in 2013 (27), Liu et al. in 2014 (29), Su et al. in 2014 (51) and Ewelt et al. in 2015 (14).

\section{OFF-LABEL USE and REPORTING on ADVERSE EVENTS}

The Italian Agency of Pharmacology (AIFA) was the first European authority that approved FL for use in neurosurgery in July 2015 (Agenza Italiana del Farmaco 'AlFA', determina n.905/2015, 15 luglio 2015). In all other countries, FL must be used off-label, and patients must preoperatively provide written informed consent. 
Two case reports have described severe adverse events after the injection of $F L$ for neurosurgical purposes $(13,52)$. Both patients underwent resection of HGG after the injection of high-dose FL, but only for 1 patient was the dose specified with $20 \mathrm{mg} / \mathrm{kg}$ bodyweight. Both patients suffered severe hypotension during general anesthesia, and, in one patient, surgery had to be discontinued. Both patients had to be admitted to the intensive care unit and fully recovered after the administration of adrenalin, prednisolone, and atropine.

We could not find any further reports on anaphylactic or severe allergic reactions in the current literature. However, all authors have described the immediate but transient yellow discoloration of the skin and the urine -due to quick renal excretion of FL- for a maximum of 24 hours.

Despite the very low risk of any severe adverse reactions due to low-dose i.v. injection of FL, neurosurgeons should be aware of any liver and kidney dysfunction, pulmonary spasm, and history of allergic reactions to contrast dye. In such cases, use of FL should be reconsidered.

\section{CURRENT CONCEPTS (PRELIMINARY DATA and ONGOING TRIALS)}

FL can be used under white light or under a dedicated surgical microscope filter. The use with white light requires doses up to $20 \mathrm{mg} / \mathrm{kg}$ bodyweight, whereas the dose can be reduced to $2-4 \mathrm{mg} / \mathrm{kg}$ under a light filter (e.g. Y560). Because we are highly experienced in using low-dose FL under filtered light, we thoroughly recommend this combination.

Fluorescence-guided surgery with FL under a dedicated surgical microscope filter is becoming increasingly established worldwide. Many neurosurgeons are deeply impressed by the possibilities and advantages of FL. First of all, the quality of the visualization of the surgical field is excellent. After a few operations, surgeons will increasingly use the filter mode because dissection and coagulation can be easily conducted without affecting the operative work flow by having to switch to the white light mode.

Another important issue is the ready availability and accessibility of FL, its very low purchase cost, and its very few adverse effects. In contrast to 5-ALA, FL does not require prevention from light, and no severe skin reactions have been described to the best of our knowledge. Furthermore, FL is completely excreted within 24 hours.

However, the efficacy of FL-guided surgery in malignant brain tumors is still discussed controversially. Since FL is a marker for BBB disruption, some authors have speculated that only unspecific changes in the tumor environment are depicted and that the lesion would not be visualized specifically enough (14). From our point of view, such concern is inappropriate because the FL-guided technique has repeatedly resulted in high rates of complete resections, particularly in HGG (as mentioned above). Notwithstanding, it is mandatory to present prospective data that prove the efficacy of the FLguided technique surgery beyond question. Such data are presently generated in the FLUOGLIO trial.
The FLUOGLIO trial (Eudra CT no. 2011-002527-18) is a multicenter phase-II trial that has been designed according to the 5-ALA trial (49) with similar primary and secondary endpoints. The primary endpoint is the rate of complete resection in the early postoperative volumetric contrastenhanced T1-weighted MR-sequence. Secondary endpoints are PFS, OS, neurological outcome, and the toxicity of FL. To date, Acerbi et al. have provided two publications with preliminary data $(2,3)$, showing a rate of complete resection in $80 \%$ of the patients with HGG.

The final evaluation and publication of the FLUOGLIO trial data are scheduled for 2016. Presumably, the results will confirm the excellent GTR rates of the retrospective series presented in this review.

A very important and promising ongoing study on the accuracy and benefit of FL-guided surgery has been outlined during the $83^{\text {rd }}$ American Association of Neurological Surgeons Annual Scientific Meeting, May 2-6, 2015. Quantifying the grade of fluorescence staining in HGG, Ung et al. concluded that fluorescein-stained tissue is highly associated with the presence of glioma tissue. By objective analytical quantification of intraoperative images with conventional photo-processing software, FL could be used to identify tumor tissue that is not shown by MR contrast imaging. Further analysis of the possibilities to digitally quantify FL fluorescence would provide novel and important insights (Ung et al, 83 ${ }^{\text {rd }}$ AANS annual scientific meeting 2015, article ID AA-30799).

\section{- FUTURE PROJECTS (FLUCEM, FLUOGLIO PHASE-III)}

To further evaluate the effect of FL under the Y560 filter, a phase trial III has been designed in which a large cohort of patients will be included. This study under Italian leadership has been planned as a multicenter, prospectively randomized, and controlled trial.

To investigate the efficacy, feasibility, and EOR of CM removed by FL-guided surgery under the Y560 filter, the authors of this article have designed an international, multicenter, prospective, and open-label trial (Fluorescein in Cerebral Metastases, 'FLUCEM').

\section{- DISCUSSION and PERSPECTIVE}

The usefulness of FL under filtered light (e.g. Y560 filter) for resecting malignant brain tumors must be verified by prospective evaluation. This issue is actually being evaluated for HGG with the participation of the authors of this article in the FLUOGLIO trial. In 2013 and 2014, two papers with preliminary data from the FLUOGLIO trial were published by the Italian group of Francesco Acerbi $(2,3)$. The trial showed a high rate of complete resection in $80 \%$ and powerful sensitivity and specificity rates of $91 \%$ and $100 \%$. The Indianapolis group of Aaron Cohen-Gadol additionally calculated the accuracy of predicting high load in histological specimens in stereotactic needle biopsies in HGG [sensitivity $79 \%$ and specificity $100 \%$ (42)] in 2014 and in 2015 [sensitivity $82.2 \%$ and specificity $90.9 \%(11)]$. 
As outlined in Tables $\mathrm{IA}$ and IB, no retrospective analysis yielded a rate of complete resection or GTR below $80 \%$, irrespective of the mode of illumination. Thus, even FL-guided surgery under white light may be assumed to significantly improve the quality of resection, not only in malignant gliomas, but also in CM, PCNSL, and skull base tumors. Similarly, however, prospective analysis is mandatory to prove the usefulness of FL in non-glioma lesions.

Presently, the literature provides much more scientific data on 5-ALA than on FL. Fluorescence-guided surgery with 5-ALA is certainly efficient and does have its place in neuro-oncologic surgery. Nevertheless, the investigation of innovative technical tools or alternative dyes is important and justified. Therefore, the continuous criticism of some 5-ALA supporters who question the rationale behind the FL-guided technique is difficult to understand $(12,48,50)$. On the other hand, many accredited neurosurgeons such as Mitchell Berger or John Sampson accompany the discussion with thoughtful editorials and recommendations $(5,15)$. In the light of the current debate, scientific process may be enhanced by including all experts in the field of modern FL-guided surgery, such as Francesco Acerbi, Peter Nakaji, and Aaron Cohen-Gadol (6).

In short, FL yields the same results as 5-ALA but in a much easier manner. FL is a readily available method for fluorescence-guided tumor resection but is a marker of contrast enhancement rather than a marker of tumors. FL may improve resection with minimal risk, and tumor margins are clearly visualized (Figure 2).

The surrogate for complete resection is the contrast-enhanced tumor in the T1-weighted MR-sequence, as recently outlined again by Hadjipanayis et al. In a review on the use of 5-ALA in HGG (17), the rates of complete resection by means of 5 -ALA ranged from $63 \%$ to $89 \%$. However, due to its high purchase costs and its limited availability, the use of 5-ALA is restricted to a few countries only. Furthermore, special equipment (surgical microscope filter) and experience is needed to conduct fluorescence-guided surgery with 5-ALA. As one of the few neurosurgical centers in the world, we justifiably claim to have sufficient experience in the use of both fluorescence techniques to assess their usefulness and practicability. We think it more practical to apply the FL-guided technique in almost all types of contrast-enhanced tumors of the brain, in which GTR should be achieved. The illumination of the Y560 filter allows continuous dissection, resection, and coagulation without switching from one light source to the other, as necessary when using the 5-ALA technique. The borders are sharply demarcated and represent the areas of MR-contrast enhancement. FL can be injected during the induction of anesthesia, does not cause any severe adverse effects, and is excreted rapidly. Furthermore, the availability of $\mathrm{FL}$ is warranted all over the world, and the costs are almost negligible.

In our opinion, FL-guided surgery provides all scientific preconditions that justify its clinical use and further evaluation in the field of neuro-oncologic surgery.

\section{ACKNOWLEDGMENT}

All authors received financial support for training purposes from Carl Zeiss Meditec (Oberkochen, Germany). Karl-Michael Schebesch and Alexander Brawanski received honoraria and travel fees from Carl Zeiss Meditec (Oberkochen, Germany).

\section{REFERENCES}

1. Abbott NJ, Ronnback L, Hansson E: Astrocyte-endothelial interactions at the blood-brain barrier. Nature reviews. Neuroscience 7(1):41-53, 2006

2. Acerbi F, Broggi M, Eoli M, Anghileri E, Cuppini L, Pollo B, Schiariti M, Visintini S, Orsi C, Franzini A, Broggi G, Ferroli $P$ : Fluorescein-guided surgery for grade IV gliomas with a dedicated filter on the surgical microscope: Preliminary results in 12 cases. Acta Neurochir 155(7):1277-1286, 2013

3. Acerbi F, Broggi M, Eoli M, Anghileri E, Cavallo C, Boffano C, Cordella R, Cuppini L, Pollo B, Schiariti M, Visintini S, Orsi C, La Corte E, Broggi G, Ferroli P: Is fluorescein-guided technique able to help in resection of high-grade gliomas? Neurosurg Focus 36(2):E5, 2014

4. Behbahaninia M, Martirosyan NL, Georges J, Udovich JA, Kalani MY, Feuerstein BG, Nakaji P, Spetzler RF, Preul MC: Intraoperative fluorescent imaging of intracranial tumors: A review. Clin Neurol Neurosurg 115(5):517-528, 2013

5. Berger MS: The fluorescein-guided technique. Neurosurg Focus 36(2):E6, 2014

6. Brawanski A, Acerbi F, Nakaji P, Cohen-Gadol A, Schebesch $\mathrm{KM}$ : Poor man-rich man fluorescence. Is this really the problem? Acta Neurochir 157(11):1959-1961, 2015

7. Chen B, Wang H, Ge P, Zhao J, Li W, Gu H, Wang G, Luo Y, Chen D: Gross total resection of glioma with the intraoperative fluorescence-guidance of fluorescein sodium. Int J Med Sci 9(8):708-714, 2012

8. da Silva CE, da Silva JL, da Silva VD: Use of sodium fluorescein in skull base tumors. Surg Neurol Int 1:70, 2010

9. da Silva CE, da Silva VD, da Silva JL: Sodium fluorescein in skull base meningiomas: A technical note. Clin Neurol Neurosurg 120:32-35, 2014

10. da Silva CE, da Silva VD, da Silva JL: Convexity meningiomas enhanced by sodium fluorescein. Surg Neurol Int 5:3, 2014

11. Diaz RJ, Dios RR, Hattab EM, Burrell K, Rakopoulos P, Sabha N, Hawkins C, Zadeh G, Rutka JT, Cohen-Gadol AA: Study of the biodistribution of fluorescein in glioma-infiltrated mouse brain and histopathological correlation of intraoperative findings in high-grade gliomas resected under fluorescein fluorescence guidance. J Neurosurg 122(6):1360-1369, 2015

12. Diez Valle R, Tejada Solis S: Answer to: "sodium fluoresceinguided resection under the YELLOW 560-nm surgical microscope filter in malignant brain tumor surgery-a feasibility study" (April 2013, volume 155, issue 4, pp 693-69). Acta Neurochir 155(7):1319-1320, 2013

13. Dilek $\mathrm{O}$, Ihsan $\mathrm{A}$, Tulay $\mathrm{H}$ : Anaphylactic reaction after fluorescein sodium administration during intracranial surgery. J Clin Neurosci 18(3):430-431, 2011 
14. Ewelt C, Nemes A, Senner V, Wolfer J, Brokinkel B, Stummer W, Holling M: Fluorescence in neurosurgery: Its diagnostic and therapeutic use. Review of the literature. J Photochem Photobiol B 148:302-309, 2015

15. Fecci PE, Babu R, Adamson DC, Sampson JH: Editorial: Turning fluorescence into black and white. J Neurosurg 122(6):1356-1358, 2015

16. Gempt J, Meyer B: Does fluorescein sodium-guided resection of cerebral metastases increase rates of total resections? Acta Neurochir 157(6):897, 2015

17. Hadjipanayis CG, Widhalm G, Stummer W: What is the surgical benefit of utilizing 5-Aminolevulinic Acid for fluorescenceguided surgery of malignant gliomas? Neurosurgery 77(5):663673, 2015

18. Hendricks BK, Cohen-Gadol AA, Miller JC: Novel delivery methods bypassing the blood-brain and blood-tumor barriers. Neurosurg Focus 38(3):E10, 2015

19. Ichioka T, Miyatake S, Asai N, Kajimoto Y, Nakagawa T, Hayashi $\mathrm{H}$, Kuroiwa T: Enhanced detection of malignant glioma xenograft by fluorescein-human serum albumin conjugate. $J$ Neurooncol 67(1-2):47-52, 2004

20. Kamp MA, Dibue M, Niemann L, Reichelt DC, Felsberg J, Steiger HJ, Szelényi A, Rapp M, Sabel M: Proof of principle: Supramarginal resection of cerebral metastases in eloquent brain areas. Acta Neurochir 154(11):1981-1986, 2012

21. Keles GE, Anderson B, Berger MS: The effect of extent of resection on time to tumor progression and survival in patients with glioblastoma multiforme of the cerebral hemisphere. Surg Neurol 52(4):371-379, 1999

22. Koc K, Anik I, Cabuk B, Ceylan S: Fluorescein sodium-guided surgery in glioblastoma multiforme: A prospective evaluation. Br J Neurosurg 22(1):99-103, 2008

23. Kuroiwa T, Kajimoto $\mathrm{Y}$, Ohta T: Development of a fluorescein operative microscope for use during malignant glioma surgery: A technical note and preliminary report. Surg Neurol 50(1):4148; discussion 48-49, 1998

24. Kuroiwa T, Kajimoto $\mathrm{Y}$, Ohta T: Surgical management for supratentorial astrocytic tumors. Minim Invasive Neurosurg 42(4):182-186, 1999

25. Lacroix M, Abi-Said D, Fourney DR, Gokaslan ZL, Shi W, DeMonte F, Lang FF, McCutcheon IE, Hassenbusch SJ, Holland E, Hess K, Michael C, Miller D: A multivariate analysis of 416 patients with glioblastoma multiforme: Prognosis, extent of resection, and survival. J Neurosurg 95(2):190-198, 2001

26. Lee J, Baird A, Eliceiri BP: In vivo measurement of gliomainduced vascular permeability. Methods in molecular biology 763:417-422, 2011

27. Li Y, Rey-Dios R, Roberts DW, Valdes PA, Cohen-Gadol AA: Intraoperative fluorescence-guided resection of highgrade gliomas: A comparison of the present techniques and evolution of future strategies. World Neurosurg 82(1-2):175185, 2014

28. Liu JG, Yang SF, Liu YH, Wang X, Mao Q: Magnetic resonance diffusion tensor imaging with fluorescein sodium dyeing for surgery of gliomas in brain motor functional areas. Chin Med $J$ (Engl) 126(13):2418-2423, 2013

29. Liu JT, Meza D, Sanai N: Trends in fluorescence image-guided surgery for gliomas. Neurosurgery 75(1):61-71, 2014
30. Marbacher S, Klinger E, Schwyzer L, Fischer I, Nevzati E, Diepers M, Roelcke U, Fathi AR, Coluccia D, Fandino J: Use of fluorescence to guide resection or biopsy of primary brain tumors and brain metastases. Neurosurg Focus 36(2):E10, 2014

31. Marko NF, Weil RJ, Schroeder JL, Lang FF, Suki D, Sawaya RE: Extent of resection of glioblastoma revisited: Personalized survival modeling facilitates more accurate survival prediction and supports a maximum-safe-resection approach to surgery. J Clin Oncol 32(8):774-782, 2014

32. Martirosyan NL, Georges J, Eschbacher JM, Cavalcanti DD, Elhadi AM, Abdelwahab MG, Scheck AC, Nakaji P, Spetzler RF, Preul MC: Potential application of a handheld confocal endomicroscope imaging system using a variety of fluorophores in experimental gliomas and normal brain. Neurosurg Focus 36(2):E16, 2014

33. Moore GE: Fluorescein as an Agent in the differentiation of normal and malignant tissues. Science 106(2745):130-131, 1947

34. Moore GE, Peyton WT, French LA, Walker WW: The clinical use of fluorescein in neurosurgery; the localization of brain tumors. J Neurosurg 5(4):392-398, 1948

35. Nduom EK, Yang C, Merrill MJ, Zhuang Z, Lonser RR: Characterization of the blood-brain barrier of metastatic and primary malignant neoplasms. J Neurosurg 119(2):427-433, 2013

36. Novotny HR, Alvis DL: A method of photographing fluorescence in circulating blood in the human retina. Circulation 24:82-86, 1961

37. Okuda T, Kataoka K, Kato A: Effectiveness of intraoperative fluorescence for diagnosis of malignant lymphoma. No Shinkei Geka 36(11):1001-1004, 2008

38. Okuda T, Kataoka K, Yabuuchi T, Yugami $H$, Kato $A$ : Fluorescence-guided surgery of metastatic brain tumors using fluorescein sodium. J Clin Neurosci 17(1):118-121, 2010

39. Oppenlander ME, Wolf AB, Snyder LA, Bina R, Wilson JR, Coons SW, Ashby LS, Brachman D, Nakaji P, Porter RW, Smith KA, Spetzler RF, Sanai N: An extent of resection threshold for recurrent glioblastoma and its risk for neurological morbidity. J Neurosurg 120(4):846-853, 2014

40. Rabb MF, Burton TC, Schatz H, Yannuzzi LA: Fluorescein angiography of the fundus: A schematic approach to interpretation. Surv Ophthalmol 22(6):387-403, 1978

41. Rey-Dios R, Cohen-Gadol AA: Technical principles and neurosurgical applications of fluorescein fluorescence using a microscope-integrated fluorescence module. Acta Neurochir 155(4):701-706, 2013

42. Rey-Dios R, Hattab EM, Cohen-Gadol AA: Use of intraoperative fluorescein sodium fluorescence to improve the accuracy of tissue diagnosis during stereotactic needle biopsy of highgrade gliomas. Acta Neurochir 156(6):1071-1075; discussion 1075, 2014

43. Sanai N, Polley MY, McDermott MW, Parsa AT, Berger MS: An extent of resection threshold for newly diagnosed glioblastomas. J Neurosurg 115(1):3-8, 2011 
44. Schebesch KM, Proescholdt M, Hohne J, Hohenberger C, Hansen E, Riemenschneider MJ, Ullrich W, Doenitz C, Schlaier J, Lange M, Brawanski A: Sodium fluorescein-guided resection under the YELLOW $560 \mathrm{~nm}$ surgical microscope filter in malignant brain tumor surgery-a feasibility study. Acta Neurochir 155(4):693-699, 2013

45. Schebesch KM, Hoehne J, Hohenberger C, Acerbi F, Broggi M, Proescholdt M, Wendl C, Riemenschneider MJ, Brawanski A. Clin Neurol Neurosurg. 2015 Dec;139:125-8.

46. Schebesch KM, Hoehne J, Hohenberger C, Proescholdt M, Riemenschneider MJ, Wendl C, Brawanski A: Fluorescein sodium-guided resection of cerebral metastases-experience with the first 30 patients. Acta Neurochir 157(6):899-904, 2015

47. Shinoda J, Yano H, Yoshimura S, Okumura A, Kaku Y, Iwama T, Sakai N: Fluorescence-guided resection of glioblastoma multiforme by using high-dose fluorescein sodium. Technical note. J Neurosurg 99(3):597-603, 2003

48. Stockhammer F: What does fluorescence depict in glioma surgery? Acta Neurochir 155(8):1479-1480, 2013
49. Stummer W, Pichlmeier U, Meinel T, Wiestler OD, Zanella F, Reulen HJ; ALA-Glioma Study Group: Fluorescence-guided surgery with 5-aminolevulinic acid for resection of malignant glioma: A randomised controlled multicentre phase III trial. Lancet. Oncology 7(5):392-401, 2006

50. Stummer W: Poor man's fluorescence? Acta Neurochir 157(8):1379-1381, 2015

51. Su X, Huang QF, Chen HL, Chen J: Fluorescence-guided resection of high-grade gliomas: A systematic review and meta-analysis. Photodiagnosis Photodyn Ther 11(4):451-458, 2014

52. Tanahashi S, Lida H, Dohi S: An anaphylactoid reaction after administration of fluorescein sodium during neurosurgery. Anesth Analg 103(2):503, 2006

53. Yoo H, Kim YZ, Nam BH, Shin SH, Yang HS, Lee JS, Zo JI, Lee $\mathrm{SH}$ : Reduced local recurrence of a single brain metastasis through microscopic total resection. J Neurosurg 110(4):730736, 2009 